\title{
Spin-isospin stability of nuclear matter
}

\author{
N. Kaiser \\ Physik-Department T39, Technische Universität München, D-85747 Garching, Germany
}

\begin{abstract}
We calculate the density-dependent spin-isospin asymmetry energy $J\left(k_{f}\right)$ of nuclear matter in the three-loop approximation of chiral perturbation theory. The interaction contributions to $J\left(k_{f}\right)$ originate from one-pion exchange, iterated one-pion exchange, and irreducible two-pion exchange with no, single, and double virtual $\Delta$-isobar excitation. We find that the approximation to $1 \pi$-exchange and iterated $1 \pi$-exchange terms (which leads already to a good nuclear matter equation of state by adjusting an emerging contactterm) is spin-isospin stable, since $J\left(k_{f 0}\right) \simeq 24 \mathrm{MeV}>0$. The inclusion of the chiral $\pi N \Delta$ dynamics, necessary in order to guarantee the spin-stability of nuclear matter, keeps this property intact. The corresponding spin-isospin asymmetry energy $J\left(k_{f}\right)$ stays positive even for extreme values of an undetermined short-distance parameter $J_{5}$ (whose possible range we estimate from realistic NN-potentials). The largest positive contribution to $J\left(k_{f}\right)$ (a term linear in density) comes from a two-body contact-term with its strength fitted to the empirical nuclear matter saturation point.
\end{abstract}

PACS: 12.38.Bx, 21.30.-x, 21.65.+f

In recent years a novel approach to the nuclear matter problem has emerged. Its key element is a separation of long- and short-distance dynamics and an ordering scheme in powers of small momenta. At nuclear matter saturation density $\rho_{0} \simeq 0.16 \mathrm{fm}^{-3}$ the Fermi momentum $k_{f 0}$ and the pion mass $m_{\pi}$ are comparable scales $\left(k_{f 0} \simeq 2 m_{\pi}\right)$, and therefore pions must be included as explicit degrees of freedom in the description of the nuclear many-body dynamics. The contributions to the energy per particle $\bar{E}\left(k_{f}\right)$ of isospin-symmetric (spin-saturated) nuclear matter as they originate from chiral pion-nucleon dynamics have been computed up to threeloop order in Refs. $[1,2]$. Both calculations are able to reproduce the empirical saturation point of nuclear matter by adjusting one single parameter (either a contact-coupling $g_{0}+g_{1} \simeq 3.23[1]$ or a cutoff scale $\Lambda \simeq 0.65 \mathrm{GeV}[2]$ ) related to unresolved short-distance dynamics. ${ }^{1}$ The basic mechanism for saturation in this approach is a repulsive contribution to the energy per particle $\bar{E}\left(k_{f}\right)$ generated by Pauli-blocking in second order (iterated) pion-exchange. As outlined in Sec. 2.5 of Ref.[2] this mechanism becomes particularly transparent by taking the chiral limit $m_{\pi}=0$. In that case the interaction contributions to $\bar{E}\left(k_{f}\right)$ are completely summarized by an attractive $k_{f}^{3}$-term and a repulsive $k_{f}^{4}$-term where the parameter-free prediction for the coefficient of the latter is very close to the one extracted from a realistic nuclear matter equation of state.

In a recent work [3] we have extended the chiral approach to nuclear matter by including systematically the effects from $2 \pi$-exchange with virtual $\Delta(1232)$-isobar excitation. The physical motivation for such an extension is threefold. First, the spin-isospin-3/2 $\Delta(1232)$-resonance

\footnotetext{
${ }^{1}$ Fitting a cutoff scale, as done in Ref.[2], must be viewed as a short-term intermediate step before an eventual full effective field theory calculation. Cutoff independence of physical observables is in fact a primary goal of effective field theory.
} 
is the most prominent feature of low-energy $\pi N$-scattering. Secondly, it is well known that $2 \pi$ exchange between nucleons with excitation of virtual $\Delta$-isobars generates the needed isoscalar central NN-attraction [4] which in phenomenological one-boson exchange models is often simulated by a fictitious scalar " $\sigma$ "-meson exchange. Thirdly, the delta-nucleon mass splitting $\Delta=293 \mathrm{MeV}$ is of the same size as the Fermi momentum $k_{f 0} \simeq 2 m_{\pi}$ at nuclear matter saturation density and therefore pions and $\Delta$-isobars should both be treated as explicit degrees of freedom. A large variety of nuclear matter properties has been investigated in this extended framework in Ref.[3]. It has been found that the inclusion of the chiral $\pi N \Delta$-dynamics is able to remove most of the shortcomings of previous chiral calculations of nuclear matter $[2,5,6,7]$. However, there remain open questions concerning the role of yet higher orders in the small momentum expansion and its "convergence". The relation of the fitted short-distance parameters [2] to those of few-nucleon systems is not clear at this moment. Also, a rigorous power counting that justifies the perturbative chiral expansion for nuclear matter has not yet been formulated. Recent work by Bogner et al. [8] based on the universal low-momentum NN-potential $V_{\text {low-k }}$ may open interesting perspectives in this direction.

Irrespective of such foundational questions it is also necessary to check various stability conditions for nuclear matter in the chiral framework. In a recent paper [9] we have analyzed spin-stability. It turned that the inclusion of the chiral $\pi N \Delta$-dynamics is essential in order to guarantee the spin-stability of isospin-symmetric nuclear matter. The truncation to fourth order terms in the small momentum expansion with interaction contributions only from $1 \pi$ - and iterated $1 \pi$-exchange is spin-unstable [9]. This statement holds independently of the regularization scheme if the contact-terms (generating contributions linear in the nucleon density) are consistent with the empirical nuclear matter bulk properties: $\bar{E}\left(k_{f 0}\right) \simeq-16 \mathrm{MeV}$ and $A\left(k_{f 0}\right) \simeq 34 \mathrm{MeV}$. Now, since a nucleon possesses four internal spin and isospin degrees of freedom one can prepare nuclear matter also in a spin-isospin mixed asymmetric configuration. The stability of nuclear matter against such correlated spin-isospin deformations is the subject of the present paper. For recent work on generalized symmetry energy coefficients in the context of phenomenological Skyrme forces, also see Ref.[10]. Analogous earlier studies within Brueckner theory using the Reid soft-core NN-potential can be found in Ref.[11] .

Let us begin with defining the spin-isospin asymmetry energy $J\left(k_{f}\right)$ of (infinite) nuclear matter. Consider this many-nucleon system in a state where the equal densities of the spinup protons $(p \uparrow)$ and the spin-down neutrons $(n \downarrow)$ have an excess over the equal densities of the spin-down protons $(p \downarrow)$ and the spin-up neutrons $(n \uparrow)$. With the help of the spin- and isospin-projection operators, $\left(1 \pm \sigma_{3}\right) / 2$ and $\left(1 \pm \tau_{3}\right) / 2$, such a spin-isospin mixed asymmetric configuration is realized by the substitution:

$$
\theta\left(k_{f}-|\vec{p}|\right) \rightarrow \frac{1+\sigma_{3} \tau_{3}}{2} \theta\left(k_{+}-|\vec{p}|\right)+\frac{1-\sigma_{3} \tau_{3}}{2} \theta\left(k_{-}-|\vec{p}|\right),
$$

in the medium insertion. ${ }^{2}$ Here, $k_{+}=k_{f}(1+\epsilon)^{1 / 3}$ and $k_{-}=k_{f}(1-\epsilon)^{1 / 3}$ (with $\epsilon$ a small parameter $)$ are different Fermi momenta, chosen such that the total nucleon density $\rho=\left(k_{+}^{3}+\right.$ $\left.k_{-}^{3}\right) / 3 \pi^{2}=2 k_{f}^{3} / 3 \pi^{2}$ stays constant. Note that Eq.(1) describes a rather peculiar asymmetric configuration of nuclear matter with equal densities of protons, neutrons, spin-up states and spin-down states: $\rho_{p}=\rho_{n}=\rho_{\uparrow}=\rho_{\downarrow}=k_{f}^{3} / 3 \pi^{2}$. The expansion of the energy per particle of spin-isospin polarized nuclear matter:

$$
\bar{E}\left(k_{+}, k_{-}\right)_{\sigma \tau-\mathrm{pol}}=\bar{E}\left(k_{f}\right)+\epsilon^{2} J\left(k_{f}\right)+\mathcal{O}\left(\epsilon^{4}\right), \quad k_{ \pm}=k_{f}(1 \pm \epsilon)^{1 / 3},
$$

\footnotetext{
${ }^{2}$ Medium insertion is a technical notation for the difference between the in-medium and vacuum nucleon propagator [2]. Effectively, it sums hole-propagation and the absence of particle-propagation below the Fermi surface $|\vec{p}|<k_{f}$.
} 

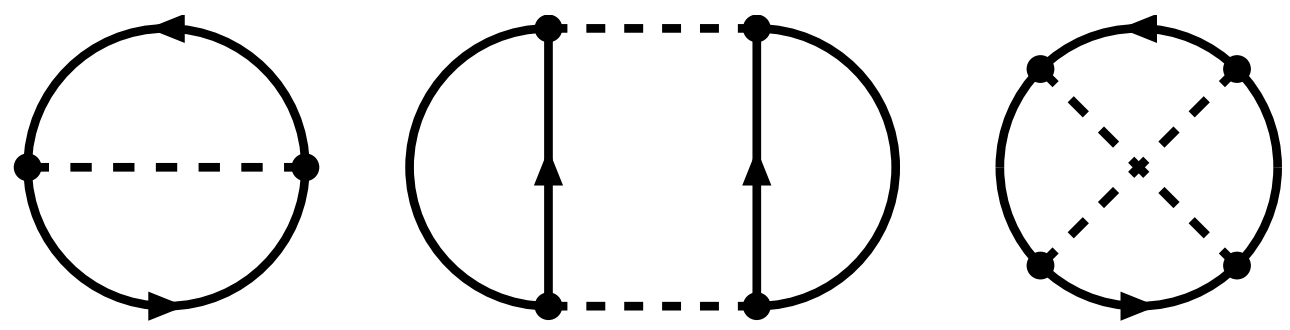

Figure 1: The two-loop one-pion exchange Fock diagram and the three-loop iterated one-pion exchange Hartree and Fock diagrams. The combinatoric factors of these diagrams are 1/2, 1/4 and $1 / 4$, in the order shown.

defines the spin-isospin asymmetry energy $J\left(k_{f}\right)$. The obvious criterion for the spin-isospin stability of nuclear matter is then the positivity of the spin-isospin asymmetry energy: $J\left(k_{f}\right)>$ 0 . The energy per particle at fixed nucleon density $\rho$ must take on its absolute minimum value in the spin- and isospin-saturated configuration.

The first contribution to the spin-isospin asymmetry energy $J\left(k_{f}\right)$ comes from the kinetic energy $\sqrt{M^{2}+p^{2}}-M$ of a non-interacting relativistic Fermi gas of nucleons:

$$
J\left(k_{f}\right)=\frac{k_{f}^{2}}{6 M}-\frac{k_{f}^{4}}{12 M^{3}},
$$

with $M=939 \mathrm{MeV}$ the (average) nucleon mass. The next term in this series, $k_{f}^{6} / 16 M^{5}$, is negligibly small at the densities of interest.

Next, we come to interaction contributions to $J\left(k_{f}\right)$. The closed in-medium diagrams related to one-pion exchange (Fock diagram) and iterated one-pion exchange (Hartree and Fock diagrams) are shown in Fig. 1. Differences in comparison to the calculation of the energy per particle $\bar{E}\left(k_{f}\right)$ in Ref.[2] occur only with respect to the factors emerging from the spin and isospin traces over closed nucleon lines and the radii $k_{ \pm}=k_{f}(1 \pm \epsilon)^{1 / 3}$ of the Fermi spheres to be integrated over. After some analytical calculation we find the following contribution to the spin-isospin asymmetry energy $J\left(k_{f}\right)$ from the $1 \pi$-exchange Fock diagram in Fig. 1 (including its relativistic $1 / M^{2}$-correction):

$$
\begin{aligned}
J\left(k_{f}\right)= & \frac{g_{A}^{2} m_{\pi}^{3}}{\left(4 \pi f_{\pi}\right)^{2}}\left\{\frac{u^{3}}{9}-\frac{u}{2}+\left(\frac{2 u}{9}+\frac{1}{8 u}\right) \ln \left(1+4 u^{2}\right)\right. \\
& \left.+\frac{m_{\pi}^{2}}{M^{2}}\left[\frac{19 u^{3}}{18}-\frac{4 u^{5}}{9}-\frac{u^{2}}{2} \arctan 2 u-\frac{u}{72}\left(1+18 u^{2}\right) \ln \left(1+4 u^{2}\right)\right]\right\} .
\end{aligned}
$$

Here, we have introduced the abbreviation $u=k_{f} / m_{\pi}$ where $m_{\pi}=135 \mathrm{MeV}$ stands for the (neutral) pion mass. As usual $f_{\pi}=92.4 \mathrm{MeV}$ denotes the weak pion decay constant and we choose the value $g_{A}=1.3$ of the nucleon axial-vector coupling constant in order to have a pionnucleon coupling constant of $g_{\pi N}=g_{A} M / f_{\pi}=13.2$. In the second and third diagram in Fig. 1 the $1 \pi$-exchange interaction is iterated (once) with itself. These second order diagrams carry the large scale enhancement factor $M$ (the nucleon mass). It stems from an energy denominator that is equal to a difference of small nucleon kinetic energies. With a medium insertion at each of two equally oriented nucleon propagators we obtain from the three-loop Hartree diagram in Fig. 1 the following contribution to the spin-isospin asymmetry energy:

$$
J\left(k_{f}\right)=\frac{\pi g_{A}^{4} M m_{\pi}^{4}}{6\left(4 \pi f_{\pi}\right)^{4}}\left\{\left(15 u+\frac{7}{2 u}\right) \ln \left(1+4 u^{2}\right)-14 u-16 u^{2} \arctan 2 u\right\} .
$$


The right Fock diagram of iterated $1 \pi$-exchange (see Fig. 1) with two medium insertions on non-neighboring nucleon propagators gives rise on the other hand to a contribution to the spin-isospin asymmetry energy of the form:

$$
\begin{aligned}
J\left(k_{f}\right)= & \frac{\pi g_{A}^{4} M m_{\pi}^{4}}{9\left(4 \pi f_{\pi}\right)^{4}}\left\{\frac{21 u}{5}-\frac{64 u^{3}}{15}-\left(9+16 u^{2}+\frac{64 u^{4}}{15}\right) \arctan u\right. \\
& +\left(\frac{33}{10 u}+\frac{14 u}{3}\right) \ln \left(1+u^{2}\right)-\left(\frac{3}{u}+2 u\right) \ln \left(1+4 u^{2}\right) \\
& \left.+\left(9-4 u^{2}\right) \arctan 2 u+\left(9+4 u^{2}\right) \int_{0}^{u} d x \frac{\arctan x-\arctan 2 x}{u\left(1+2 x^{2}\right)}\right\} .
\end{aligned}
$$

This expression does not include the contribution of a linear divergence $\int_{0}^{\infty} d l 1$ of the momentumspace loop integral. In dimensional regularization such a linear divergence is set to zero, whereas in cut-off regularization it is equal to a momentum space cut-off $\Lambda$. The additional term specific for cut-off regularization will be given in Eq.(13). An in-medium diagram with three medium insertions represents Pauli-blocking effects in intermediate NN-states induced by the filled Fermi sea of nucleons. The unequal filling of the $(p \uparrow, n \downarrow)$ and $(p \downarrow, n \uparrow)$ Fermi seas shows its consequences in the spin-isospin asymmetry energy. After some extensive algebraic manipulations we end up with the following double-integral representation of the contribution to the spin-isospin asymmetry energy $J\left(k_{f}\right)$ from the Hartree diagram in Fig. 1 with three medium insertions:

$$
\begin{aligned}
J\left(k_{f}\right)= & \frac{g_{A}^{4} M m_{\pi}^{4}}{\left(4 \pi f_{\pi}\right)^{4} u^{3}} \int_{0}^{u} d x x^{2} \int_{-1}^{1} d y\left\{\left[\frac{2 u x y\left(3 u^{2}-5 x^{2} y^{2}\right)}{\left(u^{2}-x^{2} y^{2}\right)}-\left(u^{2}+5 x^{2} y^{2}\right) H\right]\right. \\
& \times\left[\frac{2 s^{2}+s^{4}}{1+s^{2}}-2 \ln \left(1+s^{2}\right)\right]+\frac{4 u^{2} H s^{5}\left(8 s^{\prime}-9 s\right)}{9\left(1+s^{2}\right)^{2}}+\left[2 u x y+\left(u^{2}-x^{2} y^{2}\right) H\right] \\
& \left.\times\left[\left(5+s^{2}\right)\left(9 s^{2}-16 s s^{\prime}+16 s^{\prime 2}\right)+8 s\left(1+s^{2}\right)\left(2 s^{\prime \prime}-10 s^{\prime}+9 s\right)\right] \frac{s^{4}}{9\left(1+s^{2}\right)^{3}}\right\},
\end{aligned}
$$

where we have introduced several auxiliary functions:

$$
H=\ln \frac{u+x y}{u-x y}, \quad s=x y+\sqrt{u^{2}-x^{2}+x^{2} y^{2}}, \quad s^{\prime}=u \frac{\partial s}{\partial u}, \quad s^{\prime \prime}=u^{2} \frac{\partial^{2} s}{\partial u^{2}} .
$$

Note that Eq.(7) stems from a nine-dimensional principal-value integral over the product of three Fermi spheres of varying radii $k_{ \pm}=k_{f}(1 \pm \epsilon)^{1 / 3}$ which has been differentiated twice with respect to $\epsilon$ at $\epsilon=0$. Of similar structure is the contribution to $J\left(k_{f}\right)$ from the iterated $1 \pi$-exchange Fock diagram with three medium insertions. Because of the two different pion propagators in the Fock diagram one ends up (partially) with a triple-integral representation for its contribution to the spin-isospin asymmetry energy:

$$
\begin{aligned}
J\left(k_{f}\right)= & \frac{g_{A}^{4} M m_{\pi}^{4}}{72\left(4 \pi f_{\pi}\right)^{4} u^{3}} \int_{0}^{u} d x\left\{G\left(9 G_{20}+2 G_{11}+9 G_{02}-16 G_{01}-9 G\right)\right. \\
& +9 G_{10}^{2}+2 G_{01} G_{10}-5 G_{01}^{2}+4 x^{2} \int_{-1}^{1} d y \int_{-1}^{1} d z \frac{y z \theta\left(y^{2}+z^{2}-1\right)}{|y z| \sqrt{y^{2}+z^{2}-1}} \\
& \times\left[\frac{2 s^{3} t^{3}\left(16 s^{\prime} t-9 s t-12 s^{\prime} t^{\prime}\right)}{\left(1+s^{2}\right)\left(1+t^{2}\right)}+\frac{s^{2}\left[t^{2}-\ln \left(1+t^{2}\right)\right]}{\left(1+s^{2}\right)^{2}}\right. \\
& \left.\left.\times\left[\left(3+s^{2}\right)\left(16 s s^{\prime}-9 s^{2}-16 s^{2}\right)+4 s\left(1+s^{2}\right)\left(12 s^{\prime}-9 s-4 s^{\prime \prime}\right)\right]\right]\right\} .
\end{aligned}
$$


Here, we have split into factorizable and non-factorizable parts. These two pieces are distinguished by whether the (remaining) nucleon propagator in the three-loop Fock diagram can be canceled or not by terms from the product of $\pi N$-interaction vertices. The factorizable terms can be expressed through the auxiliary function:

$$
G=u\left(1+u^{2}+x^{2}\right)-\frac{1}{4 x}\left[1+(u+x)^{2}\right]\left[1+(u-x)^{2}\right] \ln \frac{1+(u+x)^{2}}{1+(u-x)^{2}},
$$

and its partial derivatives for which we have introduced a (short-hand) double-index notation:

$$
G_{i j}=x^{i} u^{j} \frac{\partial^{i+j} G}{\partial x^{i} \partial u^{j}}, \quad 1 \leq i+j \leq 2 .
$$

For the presentation of the nonfactorizable terms one needs also copies of the quantities $s$ and $s^{\prime}$ defined in Eq.(8) which depend (instead of $y$ ) on another directional cosine $z$ :

$$
t=x z+\sqrt{u^{2}-x^{2}+x^{2} z^{2}}, \quad t^{\prime}=u \frac{\partial t}{\partial u} .
$$

In the chiral limit $m_{\pi}=0$ the fourth order contributions in Eqs.(5-9) sum up to a negative $k_{f}^{4}$-term of the form: $\left.J\left(k_{f}\right)\right|_{m_{\pi}=0}=-\left(g_{A} k_{f} / 4 \pi f_{\pi}\right)^{4}(M / 405)\left(32 \pi^{2}+741+1848 \ln 2\right)$. Finally, we give the expression for the linear divergence specific to cut-off regularization:

$$
J\left(k_{f}\right)=\frac{10 g_{A}^{4} M \Lambda}{3\left(4 \pi f_{\pi}\right)^{4}} k_{f}^{3},
$$

to which only the iterated $1 \pi$-exchange Fock diagram (with two medium insertions) has contributed. In the case of the Hartree diagram the linear divergence drops out after taking the second derivative with respect to $\epsilon$. One observes that the term in Eq.(13) is just $-1 / 3$ of the corresponding contribution to the energy per particle $\bar{E}\left(k_{f}\right)$ (see Eq.(15) in Ref.[2]). In this context it is interesting to note that for terms linear in density $\rho$ the relation $3 J\left(k_{f}\right)_{\text {lin }}=-\bar{E}\left(k_{f}\right)_{\text {lin }}$ holds generally. It is a consequence of the spin-isospin structure $3-\vec{\sigma}_{1} \cdot \vec{\sigma}_{2} \vec{\tau}_{1} \cdot \vec{\tau}_{2}$ of a Fierzantisymmetric NN-contact interaction (see e.g. Eq.(35) in Ref.[12]).

Now we can turn to numerical results. In Fig. 2 we show the spin-isospin asymmetry energy $J\left(k_{f}\right)$ of nuclear matter as a function the nucleon density $\rho=2 k_{f}^{3} / 3 \pi^{2}$. The solid line corresponds to a calculation up to fourth order in small momenta. It includes besides the kinetic energy term Eq.(3) the contributions from static $1 \pi$-exchange and iterated $1 \pi$-exchange. For reasons of consistency we have dropped the small relativistic $1 / M^{2}$-correction in Eq.(4) since it is of fifth order in the small momenta $k_{f}$ and $m_{\pi}$. The cut-off scale $\Lambda=0.61 \mathrm{GeV}$ has been adjusted ${ }^{3}$ to the nuclear matter saturation point $\rho_{0}=0.173 \mathrm{fm}^{-3}$ and $\bar{E}\left(k_{f 0}\right)=-15.3 \mathrm{MeV}$. The resulting value of the nuclear matter compressibility $K=k_{f 0}^{2} \bar{E}^{\prime \prime}\left(k_{f 0}\right)=252 \mathrm{MeV}$ is consistent with a recent extrapolation from giant monopole resonances of heavy nuclei [13], which gave $K=(260 \pm 10) \mathrm{MeV}$. One can read off from Fig. 2 a positive value of the spin-isospin asymmetry energy at saturation density: $J\left(k_{f 0}\right)=J\left(2 m_{\pi}\right)=23.9 \mathrm{MeV}$. It indicates the spinisospin stability of nuclear matter in this approximation. The largest positive contribution to $J\left(2 m_{\pi}\right)=23.9 \mathrm{MeV}$ comes from the term, Eq.(13), linear in density and amounts to $59.2 \mathrm{MeV}$ at saturation density $k_{f 0}=2 m_{\pi}$. Compared to that the largest negative contribution is $-32.8 \mathrm{MeV}$ and it stems from the iterated $1 \pi$-exchange Fock diagram with two medium insertions, Eq.(6). The remaining numerically smaller contributions cancel each other to a large extent. It must

\footnotetext{
${ }^{3}$ This provisional procedure introduces a model-dependence that lies outside effective field theory.
} 


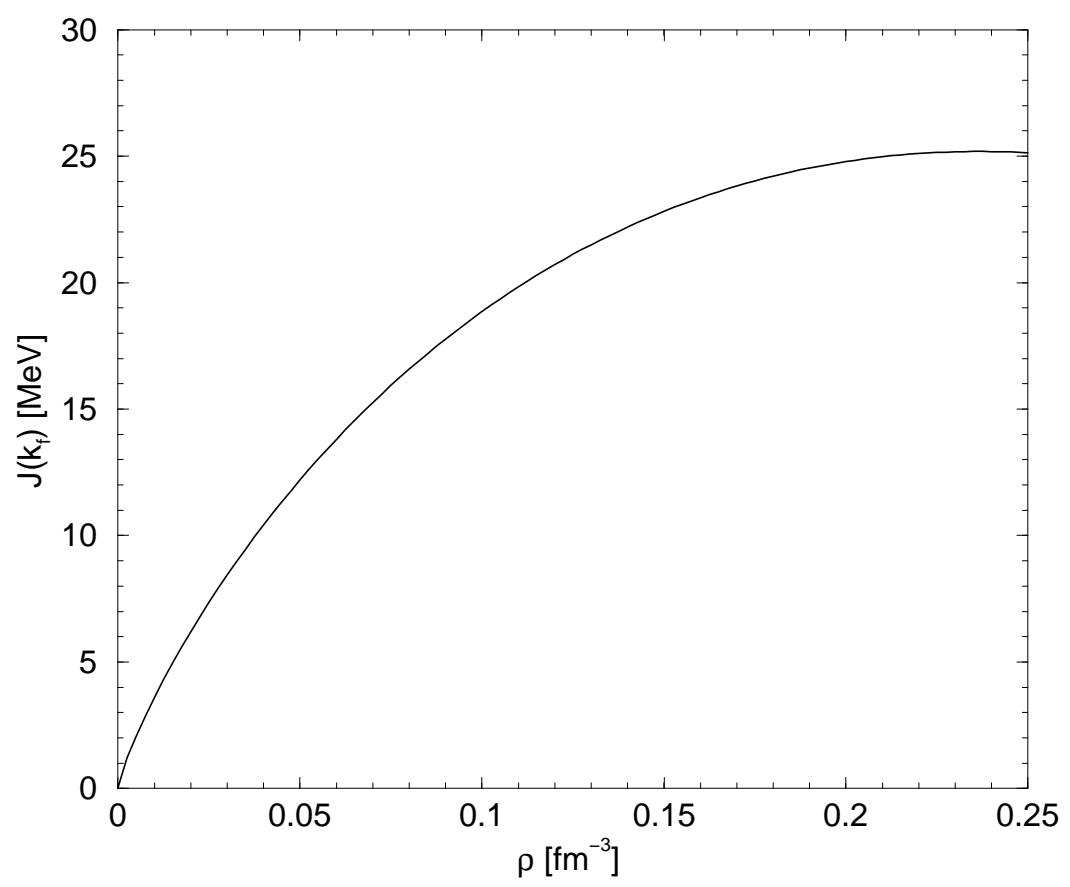

Figure 2: The spin-isospin asymmetry energy $J\left(k_{f}\right)$ of nuclear matter versus the nucleon density $\rho=2 k_{f}^{3} / 3 \pi^{2}$. The solid line shows the result of a calculation up to fourth order in small momenta including $1 \pi$-exchange and iterated $1 \pi$-exchange. The cut-off scale $\Lambda=0.61 \mathrm{GeV}$ has been adjusted to the saturation point: $\rho_{0}=0.173 \mathrm{fm}^{-3}, \bar{E}\left(k_{f 0}\right)=-15.3 \mathrm{MeV}$. The positive values of $J\left(k_{f}\right)$ indicate the spin-isospin stability of nuclear matter in this approximation.

however be stressed that at this level of approximation, with interaction terms only from $1 \pi$ exchange and iterated $1 \pi$-exchange, nuclear matter is spin-unstable [9]. The inclusion of higher order terms (in particular $2 \pi$-exchange with virtual $\Delta$-isobar excitation) is mandatory in order to achieve spin-stability of nuclear matter.

Therefore, we turn now to contributions to $J\left(k_{f}\right)$ of fifth order in the small momentum expansion. At three-loop order these terms are generated by (irreducible) two-pion exchange between nucleons. The corresponding one-loop diagrams for elastic NN-scattering are shown in Fig. 3. Since we are counting the delta-nucleon mass splitting $\Delta=293 \mathrm{MeV}$ (together with $k_{f}$ and $\left.m_{\pi}\right)$ as a small momentum scale the diagrams with single and double virtual $\Delta(1232)$ isobar excitation shown in Fig. 4 belong to the same order. By closing the two open nucleon lines of the one-loop diagrams in Figs. 3,4 to either two or one ring one gets (in diagrammatic representation) the Hartree or Fock contribution to the energy density. The Hartree contri-
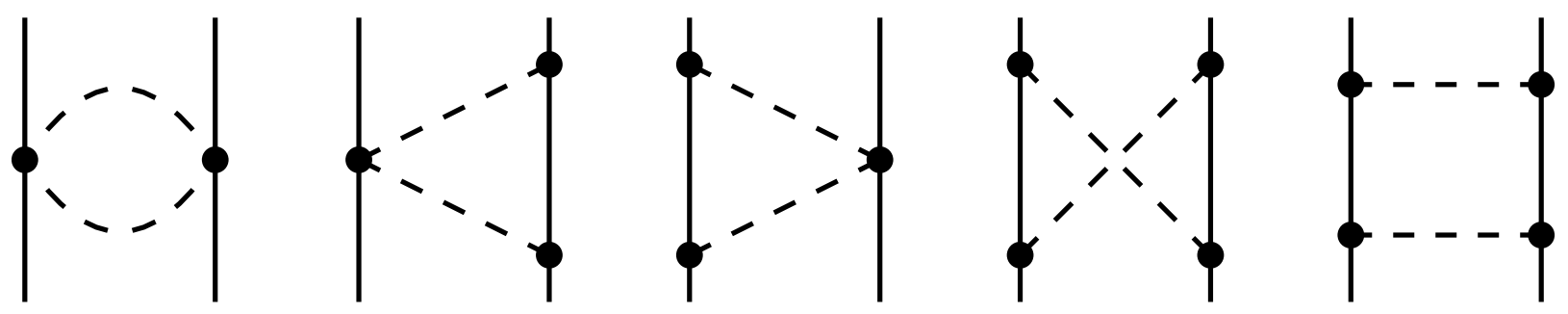

Figure 3: One-loop diagrams of irreducible two-pion exchange between nucleons. 

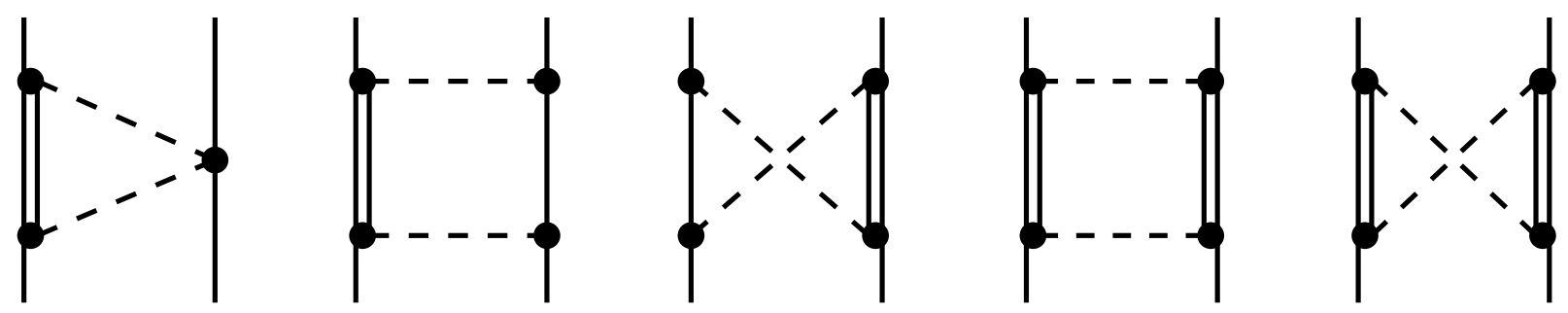

Figure 4: One-loop two-pion exchange diagrams with single and double $\Delta(1232)$-isobar excitation. Diagrams for which the role of both nucleons is interchanged are not shown.

bution to the spin-isospin asymmetry energy $J\left(k_{f}\right)$ vanishes identically because the relevant $2 \pi$-exchange NN T-matrix in forward direction is spin-independent $[4,12]$. The Fock contribution on the other hand is obtained by integrating the spin- and isospin-contracted T-matrix over the product of two Fermi spheres of radii $k_{ \pm}=k_{f}(1 \pm \epsilon)^{1 / 3}$. We separate regularization dependent short-range contributions to the T-matrix (originating from the ultra-violet divergences of the one-loop diagrams in Figs. 3,4) from the unique long-range terms with the help of a twice-subtracted dispersion relation. The occurring subtraction constants give rise to a contribution to the spin-isospin asymmetry energy of the form:

$$
J\left(k_{f}\right)=-B_{3} \frac{k_{f}^{3}}{3 M^{2}}+J_{5} \frac{k_{f}^{5}}{M^{4}} .
$$

The dimensionless parameters $B_{3}=-7.99$ has been adjusted in Ref.[3] to the saturation minimum $\bar{E}\left(k_{f 0}\right)=-16 \mathrm{MeV}$. Again, we recognize in the first part of Eq.(14) the relation $3 J\left(k_{f}\right)_{\text {lin }}=-\bar{E}\left(k_{f}\right)_{\text {lin }}$ for terms linear in the density $\rho=2 k_{f}^{3} / 3 \pi^{2}$. The other subtraction constant $J_{5}$ in front of the $k_{f}^{5} / M^{4}$-term is (a priori) not constrained by any empirical (groundstate) property of nuclear matter. The long-range parts of the $2 \pi$-exchange (two-body) Fock diagrams can be expressed as a dispersion-integral:

$$
\begin{aligned}
J\left(k_{f}\right)= & \frac{1}{6 \pi^{3}} \int_{2 m_{\pi}}^{\infty} d \mu\left\{\operatorname{Im}\left(3 W_{C}+2 \mu^{2} V_{T}+4 \mu^{2} W_{T}\right) \frac{k_{f}}{3}\left[\frac{4 k_{f}^{2}}{\mu}-\frac{8 k_{f}^{4}}{\mu^{3}}-\mu \ln \left(1+\frac{4 k_{f}^{2}}{\mu^{2}}\right)\right]\right. \\
& \left.+\operatorname{Im}\left(V_{C}+3 W_{C}+2 \mu^{2} V_{T}+6 \mu^{2} W_{T}\right)\left[\frac{\mu k_{f}}{2}-\frac{k_{f}^{3}}{\mu}+\frac{8 k_{f}^{5}}{3 \mu^{3}}-\frac{\mu^{3}}{8 k_{f}} \ln \left(1+\frac{4 k_{f}^{2}}{\mu^{2}}\right)\right]\right\},(15
\end{aligned}
$$

where $\operatorname{Im} V_{C}, \operatorname{Im} W_{C}, \operatorname{Im} V_{T}$ and $\operatorname{Im} W_{T}$ are the spectral functions of the isoscalar and isovector central and tensor NN-amplitudes, respectively. Explicit expressions of these imaginary parts for the contributions of the triangle diagram with single $\Delta$-excitation and the box diagrams with single and double $\Delta$-excitation can be easily constructed from the analytical formulas given in Sec. 3 of Ref.[4]. The $\mu$ - and $k_{f}$-dependent weighting functions in Eq.(15) take care that at low and moderate densities this spectral-integral is dominated by low invariant $\pi \pi$ masses $2 m_{\pi}<\mu<1 \mathrm{GeV}$. The contributions to the spin-isospin asymmetry energy $J\left(k_{f}\right)$ from irreducible $2 \pi$-exchange (with only nucleon intermediate states, see Fig. 3 ) can also be cast into the form Eq.(15). The corresponding non-vanishing spectral functions read [12]:

$$
\operatorname{Im} W_{C}(i \mu)=\frac{\sqrt{\mu^{2}-4 m_{\pi}^{2}}}{3 \pi \mu\left(4 f_{\pi}\right)^{4}}\left[4 m_{\pi}^{2}\left(1+4 g_{A}^{2}-5 g_{A}^{4}\right)+\mu^{2}\left(23 g_{A}^{4}-10 g_{A}^{2}-1\right)+\frac{48 g_{A}^{4} m_{\pi}^{4}}{\mu^{2}-4 m_{\pi}^{2}}\right]
$$



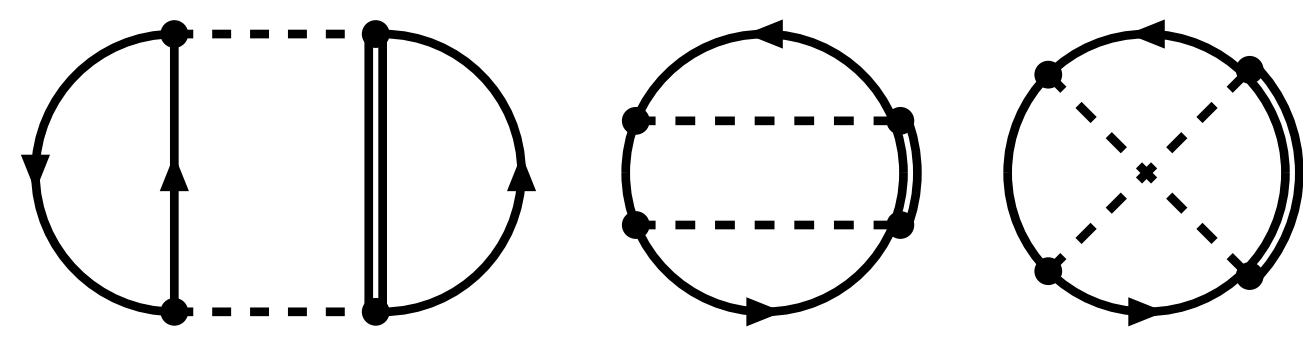

Figure 5: Hartree and Fock three-body diagrams related to $2 \pi$-exchange with single virtual $\Delta$-isobar excitation. They represent interactions between three nucleons in the Fermi sea. The combinatoric factor is 1 for each diagram.

$$
\operatorname{Im} V_{T}(i \mu)=-\frac{6 g_{A}^{4} \sqrt{\mu^{2}-4 m_{\pi}^{2}}}{\pi \mu\left(4 f_{\pi}\right)^{4}} .
$$

Next, we come to the additional $2 \pi$-exchange three-body terms which arise from Pauli blocking of intermediate nucleon states (i.e. from the $\left(1 \pm \sigma_{3} \tau_{3}\right) \theta\left(k_{ \pm}-|\vec{p}|\right)$ terms in the inmedium nucleon propagators [2]). The corresponding closed Hartree and Fock diagrams with single virtual $\Delta$-excitation are shown in Fig. 5. The contribution of the left three-body Hartree diagram to the spin-isospin asymmetry energy $J\left(k_{f}\right)$ has the following analytical form:

$$
J\left(k_{f}\right)=\frac{g_{A}^{4} m_{\pi}^{6} u^{2}}{27 \Delta\left(2 \pi f_{\pi}\right)^{4}}\left[\left(\frac{27}{4}+8 u^{2}\right) \ln \left(1+4 u^{2}\right)+2 u^{4}(1-9 \zeta)-22 u^{2}-\frac{5 u^{2}}{1+4 u^{2}}\right] .
$$

The delta propagator shows up in this expression merely via the (reciprocal) mass-splitting $\Delta=293 \mathrm{MeV}$. Furthermore, we have already inserted in Eq.(18) the empirically well-satisfied relation $g_{\pi N \Delta}=3 g_{\pi N} / \sqrt{2}$ for the $\pi N \Delta$-coupling constant. The parameter $\zeta=-3 / 4$ has been introduced in Sec. 2 of Ref.[3] in order to reduce a too strongly repulsive $\rho^{2}$-term in the energy particle $\bar{E}\left(k_{f}\right)$. It controls the strength of a three-nucleon contact interaction $\left(\zeta g_{A}^{4} / \Delta f_{\pi}^{4}\right)(\bar{N} N)^{3}$ which has the property that it contributes equally but with opposite sign to the energy per particle $\bar{E}\left(k_{f}\right)$ and the spin-isospin asymmetry energy $J\left(k_{f}\right)$. The contribution of both three-body Fock diagrams in Fig. 5 to the spin-isospin asymmetry energy $J\left(k_{f}\right)$ can be represented as:

$$
\begin{aligned}
J\left(k_{f}\right)= & \frac{g_{A}^{4} m_{\pi}^{6}}{108 \Delta\left(4 \pi f_{\pi}\right)^{4} u^{3}} \int_{0}^{u} d x\left\{-4 G_{S 01} G_{S 10}-10 G_{S 01}^{2}-18 G_{S 10}^{2}\right. \\
& +2 G_{S}\left(9 G_{S}+16 G_{S 01}-9 G_{S 02}-2 G_{S 11}-9 G_{S 20}\right)-2 G_{T 01} G_{T 10} \\
& \left.-17 G_{T 01}^{2}-9 G_{T 10}^{2}+G_{T}\left(9 G_{T}+16 G_{T 01}-9 G_{T 02}-2 G_{T 11}-9 G_{T 20}\right)\right\},
\end{aligned}
$$

with the two auxiliary functions:

$$
\begin{aligned}
& G_{S}= \frac{4 u x}{3}\left(2 u^{2}-3\right)+4 x[\arctan (u+x)+\arctan (u-x)] \\
&+\left(x^{2}-u^{2}-1\right) \ln \frac{1+(u+x)^{2}}{1+(u-x)^{2}}, \\
& G_{T}=\frac{u x}{6}\left(8 u^{2}+3 x^{2}\right)-\frac{u}{2 x}\left(1+u^{2}\right)^{2} \\
&+\frac{1}{8}\left[\frac{\left(1+u^{2}\right)^{3}}{x^{2}}-x^{4}+\left(1-3 u^{2}\right)\left(1+u^{2}-x^{2}\right)\right] \ln \frac{1+(u+x)^{2}}{1+(u-x)^{2}} .
\end{aligned}
$$




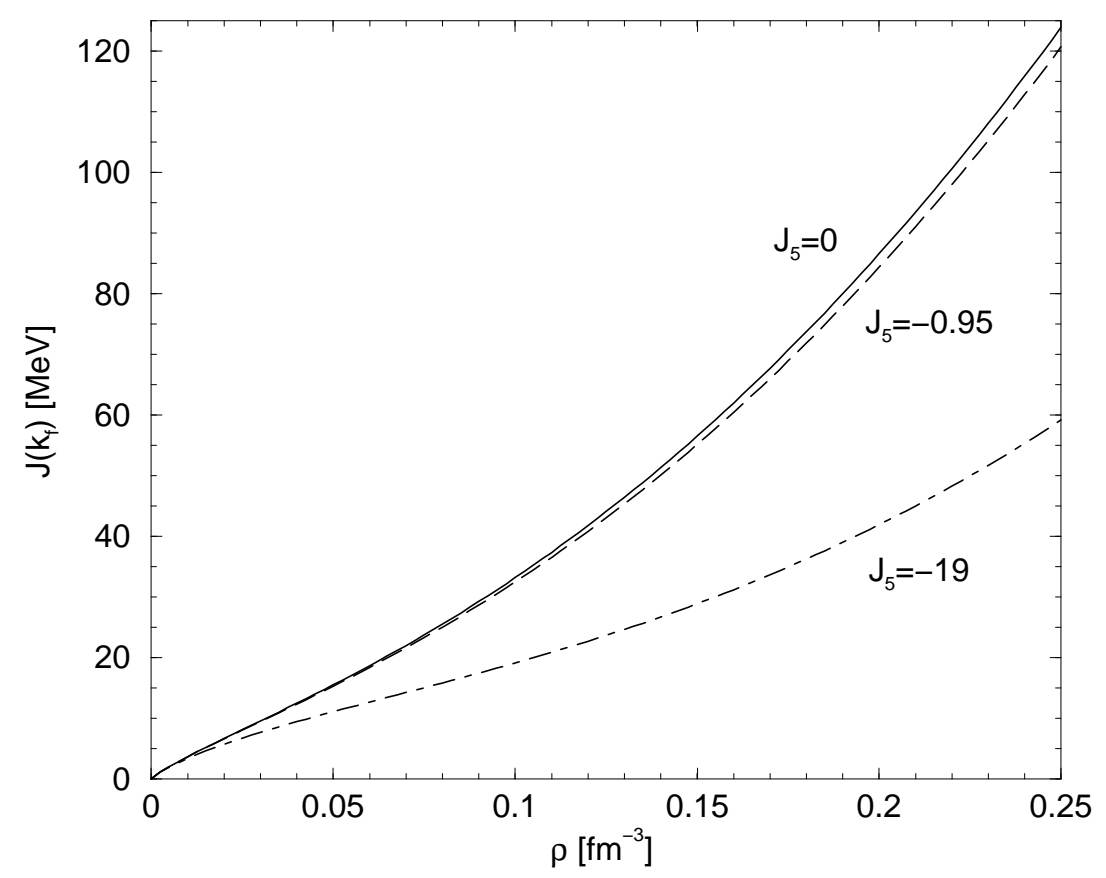

Figure 6: The spin-isospin asymmetry energy $J\left(k_{f}\right)$ of nuclear matter versus the nucleon density $\rho=2 k_{f}^{3} / 3 \pi^{2}$. In comparison to Fig. 2 the effects from $2 \pi$-exchange with single and double virtual $\Delta$-isobar excitation are now included. The solid, dashed, and dashed-dotted curves correspond to the choices $J_{5}=0,-0.95$ and -19 of the short-range parameter $J_{5}$ introduced in Eq.(14). The positive values of $J\left(k_{f}\right)$ ensure the spin-isospin stability of nuclear matter.

The double-indices on $G_{S}$ and $G_{T}$ have the same meaning as explained in Eq.(11) for the function $G$.

In Fig. 6 we show again the spin-isospin asymmetry energy $J\left(k_{f}\right)$ of nuclear matter as a function of the nucleon density $\rho=2 k_{f}^{3} / 3 \pi^{2}$. The solid line includes all the contributions from chiral $1 \pi$ - and $2 \pi$-exchange written down in Eqs.(3-9,14-19). The (yet undetermined) shortrange parameter $J_{5}$ has been set to zero, $J_{5}=0$. We note as an aside that the term linear in the density and the cut-off $\Lambda$, Eq.(13), is now not counted extra since the parameter $B_{3}=-7.99$ [3] collects all such possible terms. Numerically, these two terms linear in density are anyhow almost identical. One observes in Fig. 6 a positive spin-isospin asymmetry energy $J\left(k_{f}\right)$ which rises monotonically with the density $\rho$. The inclusion of the chiral $\pi N \Delta$-dynamics does therefore not disturb the spin-isospin stability of nuclear matter. It is also interesting to look at numerical values of $J\left(k_{f}\right)$ and their decomposition. At a Fermi momentum of $k_{f}=2 m_{\pi}$ (corresponding to $\left.\rho=0.173 \mathrm{fm}^{-3}\right)$ the spin-isospin asymmetry energy is now $J\left(2 m_{\pi}\right)=69.5 \mathrm{MeV}\left(\operatorname{setting} J_{5}=0\right)$. The most significant changes in comparison to the previous fourth order calculation come from the two-body Fock and three-body Hartree contributions Eqs. $(15,18)$ which amount together to $30.6 \mathrm{MeV}+20.7 \mathrm{MeV}=51.3 \mathrm{MeV}$. About one third thereof (namely $16.6 \mathrm{MeV}$ ) stems from the three-body contact interaction proportional to $\zeta=-3 / 4$.

The size of the short-distance parameter $J_{5}$ in Eq.(14) is still open and large negative values could endanger the spin-isospin stability. In order to get an estimate of $J_{5}$ we bring into play the complete set of four-nucleon contact-couplings written down in Eqs.(3,4) of Ref.[14]. This set represents the most general short-range $\mathrm{NN}$-interaction quadratic in momenta and it involves seven low-energy constants $C_{1}, \ldots, C_{7}$. After computing the spin-isospin asymmetry energy 
$J\left(k_{f}\right)$ from the corresponding contact-potential in Hartree-Fock approximation we find:

$$
J_{5}=\frac{M^{4}}{18 \pi^{2}}\left(C_{2}-4 C_{1}\right)=\frac{M^{4}}{144 \pi^{3}}\left[3 C\left({ }^{1} P_{1}\right)+C\left({ }^{3} P_{0}\right)+3 C\left({ }^{3} P_{1}\right)+5 C\left({ }^{3} P_{2}\right)\right] .
$$

In the second line of Eq.(22) we have reexpressed the relevant linear combination of $C_{1,2}$ through the so-called spectroscopic low-energy constants which characterize the short-range part of the NN-potential in the spin-singlet and spin-triplet $S$ - and $P$-wave states. In that representation we obtain from the entries of table IV in Ref.[14] for the three NN-potentials ${ }^{4}$ CD-Bonn, Nijm-II, and AV-18 the numbers: $J_{5}=-1.34,-0.57$, and -0.94 . The dashed line in Fig. 6 shows the spin-isospin asymmetry energy $J\left(k_{f}\right)$ which results from taking their average value $J_{5}=-0.95$. The corresponding reduction of the spin-isospin asymmetry energy is negligible. The dashed-dotted curve in Fig. 6 corresponds to the extreme choice $J_{5}=-19$. One can see that even with such a large negative $J_{5}$-value the spin-isospin stability of nuclear matter remains still preserved. We can therefore conclude that spin-isospin stability is a robust property of the chiral approach to nuclear matter (at least in the three-loop approximation). This is a important finding.

In summary we have investigated in this work the spin-isospin stability of nuclear matter in the framework of chiral perturbation theory. For that purpose we have calculated the density-dependent spin-isospin asymmetry energy $J\left(k_{f}\right)$ of nuclear matter to three-loop order. The interaction contributions to $J\left(k_{f}\right)$ originate from $1 \pi$-exchange, iterated $1 \pi$-exchange, and (irreducible) $2 \pi$-exchange with no, single, and double virtual $\Delta$-isobar excitation. We have found that the approximation to $1 \pi$ - and iterated $1 \pi$-exchange terms is spin-isospin stable, since $J\left(k_{f 0}\right)>0$. The inclusion of the chiral $\pi N \Delta$-dynamics (necessary to ensure the spinstability [9] of nuclear matter) keeps this property intact. The largest positive contribution to $J\left(k_{f}\right)$ comes from a two-body contact interaction with its strength fitted to the empirical nuclear matter saturation point.

\section{References}

[1] M. Lutz, B. Friman, and Ch. Appel, Phys. Lett. B474, 7 (2000).

[2] N. Kaiser, S. Fritsch and W. Weise, Nucl. Phys. A697, 255 (2002); and references therein.

[3] S. Fritsch, N. Kaiser, and W. Weise, Nucl. Phys. A750, 259 (2005).

[4] N. Kaiser, S. Gerstendörfer and W. Weise, Nucl. Phys. A637, 395 (1998).

[5] N. Kaiser, S. Fritsch and W. Weise, Nucl. Phys. A700, 343 (2002).

[6] S. Fritsch, N. Kaiser and W. Weise, Phys. Lett. B545, 73 (2002).

[7] S. Fritsch and N. Kaiser, Eur. Phys. J. A17, 11 (2003).

[8] S.K. Bogner, A. Schwenk, R.J. Furnstahl and A. Nogga, "Is nuclear matter perturbative with low-momentum interactions?", nucl-th/0504043.

[9] N. Kaiser, Phys. Rev. C70, 054001 (2004).

[10] F.L. Braghin, Int. J. Mod. Phys. E12, 755 (2003); Phys. Rev. C71, 064303 (2005).

[11] J. Dabrowski and P. Haensel, Phys. Rev. C7, 916 (1973); and references therein.

[12] N. Kaiser, R. Brockmann and W. Weise, Nucl. Phys. A625, 758 (1997).

[13] D. Vretenar, T. Niksic and P. Ring, Phys. Rev. C68, 024310 (2003).

[14] E. Epelbaum, Ulf-G. Meißner, W. Glöckle, and C. Elster, Phys. Rev. C65, 044001 (2002); and references therein.

\footnotetext{
${ }^{4}$ The short-distance structure of realistic NN-potentials and effective field theory could be very different. The idea here is simply to explore the extreme possible range of $J_{5}$.
} 